\title{
La conduite sociale d'un professeur de français dans une petite ville espagnole des années 1860
}

Manuel Bruña Cuevas

\section{(2) OpenEdition \\ Journals}

Édition électronique

URL : https://journals.openedition.org/dhfles/1816

DOI : $10.4000 /$ dhfles. 1816

ISSN : 2221-4038

Éditeur

Société Internationale pour l'Histoire du Français Langue Étrangère ou Seconde

Édition imprimée

Date de publication : 1 juin 2005

ISSN : 0992-7654

Référence électronique

Manuel Bruña Cuevas, «La conduite sociale d'un professeur de français dans une petite ville

espagnole des années 1860 ", Documents pour l'histoire du français langue étrangère ou seconde [En

ligne], 33/34 | 2005, mis en ligne le 01 janvier 2012, consulté le 27 mai 2021. URL : http://

journals.openedition.org/dhfles/1816 ; DOI : https://doi.org/10.4000/dhfles.1816

Ce document a été généré automatiquement le 27 mai 2021.

(c) SIHFLES 


\title{
La conduite sociale d'un professeur de français dans une petite ville espagnole des années 1860
}

\author{
Manuel Bruña Cuevas
}

\section{Introduction}

Le 12 septembre 1865, le directeur du lycée de Huelva envoie au recteur de l'université de Séville une plainte formelle contre Joseph Orfois, le professeur de français du lycée ${ }^{1}$. Cette affaire reflète ce qu'on attendait d'un professeur de l'enseignement secondaire dans l'Espagne de l'époque, tant du point de vue social que du point de vue académique. Son analyse nous aidera également à nous construire une représentation du processus de professionnalisation et d'institutionnalisation du français en Espagne au XIX ${ }^{e}$ siècle.

2 Huelva était à l'époque une petite ville faisant partie de l'académie de Séville. Les enseignements secondaire et universitaire se trouvant à l'époque étroitement liés, le recteur de l'université de Séville était la plus haute autorité dont dépendait le lycée de Huelva. Quant à la situation politique de l'Espagne, le règne d'Isabelle II touchait à sa fin. La droite monopolisait le pouvoir et un concordat signé avec le Vatican, en 1851, avait permis aux gouvernements conservateurs de s'attirer l'appui de l'Église espagnole et à celle-ci de renforcer son influence sur certains secteurs sociaux. La Constitution en vigueur (celle de 1845) définissait à nouveau l'État espagnol comme catholique et les articles 295 et 298 de la loi d'instruction publique (loi Moyano, 1857), recueillant ce qu'établissait l'article 3 du Concordat, reconnaissaient aux évêques le droit de regard sur l'enseignement, aussi bien dans le domaine des idées que dans celui de la morale et de la conduite des professeurs (Rueda, $1996: 49$ ). 


\section{Les accusations contre Orfois}

3 La plainte du directeur du lycée de Huelva contre le professeur Orfois s'est produite lorsque celui-ci y avait déjà assuré les cours de français pendant trois années. Il avait été embauché en janvier 1863 par le directeur lui-même et, grâce aux avis favorables de ce dernier et des autorités civiles de Huelva, il obtient du Ministère, en décembre 1863, la transformation de son poste intérimaire en poste de titulaire (catedrático). Ce n'est donc qu'au cours des années 1864 et 1865 que les rapports entre le directeur et Orfois ont dû passer de la cordialité à l'inimitié déclarée. Mais il y a un fait précis qui a déclenché la crise définitive. Le voici.

4 En 1865, le préfet de Huelva demande au directeur du lycée de dresser la liste des professeurs qui atteignaient le seuil de huit cents escudos de revenus ${ }^{2}$. Quoique conscient que sa collaboration avec le préfet frôlait l'illégalité, le directeur se décide à lui fournir les noms demandés. Or, Joseph Orfois ne figurait pas sur sa liste.

5 Le cas d'Orfois était délicat. D'une part, n'ayant pas à sa charge un grand nombre de cours, ses appointements se réduisaient à six cents escudos, ce qui justifiait son exclusion des listes électorales. Mais, d'autre part, Orfois n'en était pas moins professeur titulaire du lycée, et, à ce titre, il se sentait le droit d'être électeur ; le fait de l'exclure des listes électorales revenait donc, pour lui, à rabaisser son prestige social. Par conséquent, il commence un combat pour son droit de vote : le 31 août 1865, il déclare publiquement, devant le secrétaire de la mairie, que le directeur du lycée ne l'a pas inclus dans les listes par animadversion et que le préfet lui a assuré n'avoir jamais suggéré au directeur le critère des huit cents escudos. Celui-ci, se sentant diffamé, entreprend diverses démarches pour défendre son honneur; finalement, Orfois ne lui ayant toujours pas présenté ses excuses vers la mi-septembre, il dépose devant le recteur sa première plainte en neuf ans comme directeur du lycée de Huelva. Il y demande l'assignation à Orfois d'un poste dans un lycée différent, vu que le retour aux relations cordiales entre le professeur de français et le directeur semble désormais impossible.

6 Le fait que le directeur considère comme le point fondamental justifiant la mutation d'Orfois la perte de la bonne entente entre eux révèle déjà le caractère fortement hiérarchisé des relations académiques de l'époque. Toutefois, pour mieux étayer sa demande, le directeur exprime d'autres sujets de plainte contre Orfois, fort révélateurs aussi de la mentalité du moment en ce qui concerne la conduite que devait avoir un professeur d'un lycée de province.

7 Le directeur l'accuse d'avoir eu un différend avec un autre professeur du lycée et d'avoir tenu des propos méprisants pour le corps professoral, pour les Andalous et pour les Espagnols en général, ce qui renforçait sa présentation d'Orfois comme un individu qui dérangeait la cordialité devant régner dans un établissement scolaire. Et, dans cette même ligne, mais avec la nuance de mise question des décisions prises par son supérieur, le directeur l'accuse d'avoir critiqué dans son dos son refus de lui accorder une augmentation du nombre de matières à assurer. Orfois, en effet, avait voulu avoir à sa charge, en plus des cours de Français, les pratiques d'Arithmétique et de Géométrie, assignées à un professeur de l'École normale; mais le directeur fondait son refus sur les ordres qu'il avait reçus des autorités supérieures. Orfois a dès lors prétendu être nommé, comme le reste des titulaires du lycée, aux jurys d'autres matières que la sienne ; il voulait, plus précisément, pouvoir juger les examens de Mathématiques et de 
Géographie. Mais le directeur ne le lui a jamais permis parce que Orfois ne disposait d'aucun diplôme prouvant qu'il avait les connaissances nécessaires pour être membre de ces jurys. Cela revient à dire qu'Orfois, contrairement à d'autres professeurs du lycée, n'avait même pas obtenu le diplôme de baccalauréat, la loi, en effet, permettant aux professeurs de langues étrangères de ne pas l'avoir, certainement parce qu'il n'avait pas toujours été facile d'en trouver en nombre suffisant.

Outre cette ligne argumentative sur le caractère difficile d'Orfois et sur son incompatibilité avec ses collègues - notamment avec le directeur -, celui-ci a recours à deux autres sortes de raisons pour demander sa mutation : des raisons sociales et des raisons académiques.

Selon le directeur, la conduite publique du professeur de français l'a fait déchoir de l'estime des ses concitoyens. Le comportement public d'un professeur dans une petite ville de province devait constituer un modèle pour ses habitants. L'un des traits saillants de ce comportement exemplaire était, pour les autorités de l'époque, la pratique religieuse. Or, comme Orfois était un catholique pratiquant, le directeur l'accuse de l'être d'une façon si excessive qu'il provoque le rire des citoyens et des élèves : son propre tempérament ou sa méconnaissance des coutumes espagnoles, étant donné sa condition d'étranger, rendraient compte, selon le directeur, de cet état de faits. Il en donne comme exemple le fait que, lorsque Orfois se dispose à communier, il asperge préalablement d'eau de Cologne l'autel, les objets de culte et le prêtre luimême.

10 Même si ce fait suffirait à le faire apparaître aux yeux de tous, et particulièrement aux yeux des élèves, comme quelqu'un de grotesque, le plus grave c'est que ce comportement religieux pourrait n'être que de la tartuferie, vu que, d'autre part, Orfois aime à boire, ce qui n'est pas compatible avec les bonnes habitudes qui doivent orner un bon catholique. Une fois, le délégué départemental du Ministère d'Économie ${ }^{3}$ l'a surpris en état d'ivresse sur la plage d'une île proche de Huelva, et quotidiennement, après le déjeuner, il va boire du vin dans une taberna. Or, comme en France, les couches moyennes des villes espagnoles ne fréquentaient plus les tavernes; leur vie sociale se déroulait dans les círculos (cercles) et les casinos (clubs), dont les membres devaient payer une cotisation qui assurait la séparation des classes sociales. Un catedrático qui fréquentait les tabernas ruinait donc son prestige social et celui de ses collègues et représentait un mauvais exemple pour les élèves du lycée, pour les fils des couches sociales aisées. Le directeur expose que, ayant admonesté Orfois pour ses visites aux tabernas, le professeur lui avait répondu que boire du vin était pour lui une nécessité et qu'il ne pouvait en consommer en privé parce qu'il était logé dans une pension où il ne lui était pas possible d'en avoir.

11 Après avoir attaqué la tendance d'Orfois à la médisance, sa tendance à mettre en question l'autorité académique et sa conduite sociale, le directeur l'accuse, enfin, d'être un mauvais professeur. Il aurait frappé quelques élèves et le directeur lui-même l'aurait vu conduire un enfant par le cou. Mais il l'accuse surtout de ne pas parvenir à transmettre un niveau acceptable de français à ses élèves, raison pour laquelle ceux-ci n'obtiennent que des notes médiocres aux examens devant jury. Le directeur attribue ce bas niveau, d'une part, au fait qu'Orfois aurait perdu son crédit auprès des élèves par sa conduite sociale, et, de l'autre, au fait que sa méthode serait inadéquate : il s'entêtait à pratiquer un enseignement théorique, oubliant ainsi, comme le directeur le lui avait fait remarquer, que le but d'un professeur de français devait être celui d'amener ses 
élèves, sinon à parler cette langue, du moins à pouvoir la traduire. Cette remarque du directeur a plus de portée qu'elle n'a l'air. Pour l'enseignement du français aux lycées, le décret du ministre Cervera (1861) enjoignait à employer une méthode «non pas théorico-générale mais particulière et pratique » (Fernández Fraile \& Suso López, 1999 : 247), propre à préparer les élèves à l'épreuve devant jury consistant en une traduction. Le directeur, par conséquent, accusait Orfois de tenir toujours à des méthodes considérées comme dépassées et expressément défendues dans les dispositions ministérielles.

\section{La position du Conseil départemental d'instruction}

$12 \mathrm{Au}$ vu de ces graves accusations, le recteur de l'Université de Séville les présente devant le Conseil universitaire, qui croit nécessaire, avant d'arrêter une décision, de demander au Conseil d'instruction du département de Huelva son opinion sur les différents points qui constituent la plainte du directeur. Le Conseil d'instruction, à son tour, charge une commission, composée par trois de ses membres, de l'enquête correspondante et de la rédaction d'un rapport. Celui-ci a été fait et il a été assumé par le Conseil d'instruction. Curieusement, on y perçoit une volonté nette de nuancer ou de nier le bien-fondé des accusations du directeur contre Orfois.

La commission constate, en effet, qu'Orfois en veut au directeur, mais elle ne manque pas de recueillir aussi, sans la nier, l'affirmation du professeur selon laquelle son supérieur, pour sa part, «manque d'affection, d'amitié et de considération » envers lui. Elle confirme, de même, qu'il y a eu un différend entre Orfois et un autre professeur, mais ne pense pas, comme le disait le directeur, que ce fait puisse contribuer à troubler dorénavant l'harmonie dans le lycée, étant donné que ce dernier professeur n'y exerce plus ses fonctions.

Pour ce qui est d'Orfois comme enseignant, la commission avoue qu'elle n'a pu trouver, malgré ses efforts pour y parvenir, aucune preuve de mauvais traitements, mais qu'elle peut assurer, en revanche, que, contrairement à ce que soutient le directeur, certains élèves d'Orfois ont eu de très bonnes notes aux examens devant jury, en preuve de quoi elle joint à son rapport un relevé des notes obtenues par tous ses élèves des années 1863-1864 et 1864-1865.

Le troisième groupe d'imputations, relatives au mauvais comportement social d'Orfois, est celui qui mérite le plus d'attention dans le rapport de la commission. Deux seuls des faits de cet ordre avancés par le directeur n'y sont pas considérés comme faux, fort probablement parce que les rejeter serait revenu à accuser de mensonge le préfet et le délégué du Ministère d'Économie. La commission admet donc, car le préfet peut le confirmer, qu'Orfois a contesté son exclusion des listes électorales et que ses manières n'ont pas été des plus correctes, mais elle considère finalement que l'on doit pardonner à un étranger, méconnaissant les coutumes espagnoles, l'affaire en question. Quant au fait qu'Orfois ait été vu dans une île en état d'ivresse, la commission veut bien le croire puisque c'est le délégué ministériel que le directeur présente comme témoin, mais elle n'a pu le confirmer du fait que ce témoin n'exerce plus ses fonctions à Huelva, mais à Séville. Elle rejette catégoriquement, par contre, l'attribution à Orfois du «vice de l'ivresse ", vu que tous les habitants de Huelva interrogés ont nié que ce soit vrai. Et la commission d'ajouter : 
Il est vrai qu'on le voit rentrer habituellement dans une épicerie, mais il n'y va que pour prendre une tasse de café à deux sous, car il n'a pas les moyens de fréquenter un cercle ou un club, réservés, comme on le sait, aux plus fortunés. pas avoir accédé à augmenter la charge horaire d'Orfois pour lui permettre de s'intégrer pleinement dans le milieu social des classes moyennes. C'est, en effet, par la juste prétention d'Orfois d'améliorer sa situation économique que la commission justifie son insistance à demander au directeur la responsabilité de nouveaux cours ou son désir de participer à des jurys autres que ceux de Français; et c'est en invoquant aussi bien sa pauvreté que, de nouveau, sa condition d'étranger qu'elle cherche à rendre compte du fait qu'il ait pu être l'objet de quelques railleries :

En ce qui concerne l'affirmation selon laquelle Orfois aurait perdu sa réputation par sa conduite publique, et quant au fait que les gens se moqueraient de lui, cette Commission se réjouit de pouvoir assurer qu'il s'agit là d'une charge sans fondement. Sa pauvreté, la figure caractéristique de son pays et le fait qu'il n'a pas les moyens de s'habiller richement de par son très maigre traitement et parce qu'il a un fils qu'il éduque et dont il paie les études, ce sont des causes, ce sont des raisons pour le respecter plutôt que pour le condamner, ainsi que des excuses suffisantes pour son malaise continu et pour ses désirs de se voir proposer des travaux lui permettant de gagner davantage.

ces prémisses, la conclusion de la commission ne pouvait être que celle-ci : « Cette Commission conclut en réitérant que, à son avis, Orfois n'est que malheureux et non pas coupable. » Comme mesures à prendre, elle se borne à recommander la désignation d'une personne autorisée qui conseille à Orfois la façon d'éviter à l'avenir de nouveaux désaccords. Le président du Conseil d'instruction se propose lui-même pour cette tâche dans la lettre de présentation qui accompagne le rapport de la commission, envoyé au recteur une fois assumé par le Conseil.

\section{La réaction d'Orfois}

Rien ne montre dans les documents que nous avons commentés qu'Orfois ait été au courant de l'existence d'une plainte contre lui. Lorsqu'il l'apprend, vers le mois d'avril 1866 , il demande au recteur de convoquer une rencontre entre le directeur et lui pour contraster leurs points de vue sur les faits. Mais, avec cette pétition, Orfois transgressait le cours établi pour les affaires de ce genre : il aurait dû, selon les normes, soit attendre le verdict du recteur, soit, à la limite, s'adresser d'abord au Conseil d'instruction. Celui-ci ne le lui pardonnera pas.

Quand le recteur reçoit la pétition d'Orfois, il la renvoie au Conseil d'instruction, qui se voit donc obligé de fournir, le 4 mai, un nouveau rapport sur l'affaire. Or si le premier, daté d'une quinzaine de jours avant, était tout favorable au professeur, dans le second les conseillers ont complètement changé d'avis: la pétition d'Orfois, disent-ils, leur révèle enfin le vrai caractère du professeur et donne raison à la plainte présentée par le directeur. Comme on le voit, la violation par Orfois du principe de hiérarchie et d'autorité mérite, pour le Conseil, l'adoption de mesures plus sévères. 


\section{Orfois se défend} universitaire, celui-ci décide de créer, lui aussi, une commission pour étudier l'affaire ; elle était formée des directeurs respectifs du lycée et de l'école normale de Séville. Après avoir reçu la pétition d'Orfois et le second rapport, cette commission croit bon d'écouter le professeur avant de prendre position : le caractère contradictoire des deux rapports du Conseil l'a probablement poussée à s'en méfier. Le 20 mars 1867, le recteur transmet donc à Orfois, par l'intermédiaire du directeur du lycée de Huelva, déjà différent de celui qui avait présenté la plainte, les imputations dont il avait été l'objet, lui accordant huit jours pour présenter sa défense par écrit. La réponse rédigée par le professeur et datée du 28 mars offre également un bon portrait de la société de son temps.

Orfois commence par justifier son désir d'assumer plusieurs matières par la situation économique où il se trouvait à l'époque. Il précise qu'il ne comprenait pas que des professeurs de l'école normale puissent donner certains cours au lycée alors qu'il aurait pu s'en charger lui-même, mais qu'il n'a plus insisté sur ce fait dès qu'il a su que c'était ainsi par ordre direct du recteur. Il ajoute, toutefois, que sa pétition était raisonnable dans la mesure où le directeur lui-même avait à sa charge quatre matières, dont il n'avait presque pas le temps de s'occuper à cause de ses fonctions administratives; dans la mesure aussi où le secrétaire du lycée avait deux matières et dans la mesure où un autre professeur en avait également deux qu'il réunissait dans une même tranche horaire. Il admet, d'autre part, qu'il ne pouvait prouver par un diplôme qu'il possédait la formation nécessaire pour faire partie des jurys de Géographie et de Mathématiques, mais il signale que le directeur savait bien qu'il avait assez de connaissances sur ces matières pour pouvoir en juger les épreuves. Finalement, quant à sa dispute avec un collègue, il préfère ne mentionner aucun nom pour éviter que quelqu'un d'autre ne se voie mêlé à l'affaire.

En ce qui concerne son travail en classe, Orfois aborde de façon différente les deux imputations qui lui ont été faites. Pour ce qui est de sa méthode d'enseignement du français et des résultats de ses élèves aux examens, il se limite à signaler que les programmes de ses cours sont déposés, comme prévu, au secrétariat du lycée et que les notes des examens ont été publiées : les uns et les autres sont donc à la disposition des autorités et lui n'a rien d'autre à commenter là-dessus. C'est bien dommage pour nous, car nous aurions aimé le voir défendre une méthode d'enseignement du français décriée par le directeur et connaître son opinion sur le taux de réussite de ses élèves. Il est plus loquace, par contre, en ce qui concerne l'accusation de mauvais traitements :

Quand je me suis chargé de cette matière, les cours avaient lieu le soir : peu de lumière donc dans la salle de classe et aucune dans les couloirs, aussi bien à l'entrée qu'à la sortie. D'autre part, les toilettes se trouvaient dans l'aile opposée du bâtiment. Or, l'un des enfants s'est mis en tête de sortir et rentrer tous les jours à chaque instant, et, le lui ayant interdit, surtout pour éviter qu'il ne lui arrive malheur, comme en réalité il ne voulait que se promener, il a décidé, puisque je ne le laissait pas sortir, de lâcher de l'eau tous les soirs dans la salle de classe. Prévenu de son intention, je l'ai saisi sur-le-champ et, sans trop garder la mesure dans mes paroles et moins encore dans mes actes, je ne sais si je lui ai tiré sa petite oreille ou si je lui ai donné une gifle, mais je sais bien qu'il n'en a pas pour autant pleuré [...]

Documents pour l'histoire du français langue étrangère ou seconde, 33/34 | 2005 
enfin, consacre la plupart de sa défense, comme c'était le cas dans les autres documents, à réfuter les imputations ayant trait à sa conduite sociale. Contre l'accusation de boire en excès, il avoue qu'il boit, mais comme le font « les personnes bien élevées et de bonnes coutumes, et pas plus ». Il nie l'incident de la plage, mais il ajoute que, en supposant qu'il ait été vu avec un verre de plus, il faudrait se demander pourquoi le directeur, au lieu de dénoncer le fait à ce moment-là, avait attendu trois ans pour le faire.

Toujours sur le même sujet, il rejette qu'il éprouve une nécessité "absolue » de boire et, surtout, que ses visites aux tavernes soient une «habitude »; il ne peut avoir cette "habitude» parce qu'il a souvent passé des journées entières sans manger pour pouvoir nourrir son fils. Comme on le voit, le manque de ressources revient toujours dans son discours en tant que ligne argumentative :

Mais, quand il me fallait prendre un verre de vin ou de liqueur ou une tasse de café, je le faisait, selon les cas, là où je les trouvait à meilleur marché, quoique toujours dans des lieux fréquentés, alors et aujourd'hui, par des personnes de bien, telles que quelques professeurs; j'y allais avec eux et eux avec moi, chacun quand il le pouvait; mais toujours avec retenue, avec beaucoup de retenue, Monsieur. Le directeur lui-même en est sans doute au courant, bien que, plus tard, moi seul ait été tourmenté pour cela et même pour fumer, jamais les autres ni pour la même chose ni pour d'autres choses pareilles; dont je connais la raison, pouvant en rendre compte si je veux, Monsieur.

En ce qui touche aux listes électorales, il est évident qu'Orfois préfère ne pas remuer la question, s'en justifiant par le fait que, s'il apportait des preuves de la mauvaise foi du directeur dans la confection des listes, celui-ci encourrait des poursuites pénales. Mais il ajoute une phrase énigmatique : il fait remarquer que le directeur du lycée n'est plus le même et signale que le préfet de Huelva pourrait expliquer la raison pour laquelle l'ancien directeur "n'est pas sorti de cette ville avec un accompagnement amusant ». En est-il peut-être sorti accompagné de la police?

e rapport de la commission du Conseil d'instruction avait eu recours plusieurs fois à la condition d'étranger d'Orfois pour justifier son comportement. Celui-ci, en revanche, certainement parce qu'il n'avait pu lire le rapport, cherche à prouver par tous les moyens qu'il est pleinement espagnol. Il déclare même que, si on l'y oblige, il est prêt à fournir dans ce sens le témoignage

de plusieurs Ministres de la Couronne, y compris celui de Monsieur le Premier Ministre; et aussi celui de plusieurs Archevêques et révérends Évêques, celui de plusieurs Recteurs, Ambassadeurs (sauf celui de France), Préfets, Députés, Généraux; celui de beaucoup d'excellents collègues et Directeurs; celui de maints pauvres et maints détenus, et, enfin, celui de tous ceux qui m'ont vu parcourir presque toute l'Espagne, par terre et par mer, depuis un peu plus de trente ans. Certes, Monsieur, je suis pauvre ; mais j'ai toujours gardé la tête haute, le front sans tache et l'esprit tranquille, même si j'ai le coeur quelque peu agité en ce moment.

À remarquer dans cette déclaration, outre son caractère fanfaron - il en a l'air, du moins -, le temps qu'Orfois dit avoir résidé en Espagne: un peu plus de trente ans. Comme, dans le premier écrit qu'il adresse au recteur en 1866, il déclare être âgé de cinquante ans et en avoir consacré vingt-et-un au professorat, on peut établir qu'il a dû arriver en Espagne vers 1836 à l'âge de vingt ans, qu'il a commencé à donner des cours vers ses vingt-neuf ans et que son fils est né en territoire espagnol. D'autre part, s'il est vrai que la législation espagnole permettait à l'époque que les professeurs de langues étrangères des lycées ne possèdent pas la nationalité espagnole, Orfois devait bien 
l'avoir, étant donné qu'il a réclamé son inclusion dans les listes électorales. À ses cinquante ans, après avoir passé plus longtemps en Espagne qu'en France, on peut bien le croire lorsqu'il proclame qu'il se sent espagnol. Mais cela nous mène également à avoir le soupçon que, quand la commission de Huelva recourt dans son rapport à sa condition d'étranger, elle ne cherche en réalité qu'à le justifier face aux imputations du directeur, soit pour éviter sa mutation, soit dans l'intention de nuire à la réputation de celui-ci. Quoi qu'il en soit, si elle se permet de parler d'Orfois comme d'un étranger, c'est parce que, même s'il connaissait bien les coutumes du pays, il devait y avoir en lui quelque chose qui sortait du commun: un caractère bizarre, un visage au teint peu latin ou tirant trop au rouge, un accent français lorsqu'il parlait en espagnol... Réunissant tous ou certains de ces traits, donnant des cours de français et n'ayant pas d'attaches familiales à Huelva, il est fort probable qu'il y passait pour un étranger. Peutêtre en profitait-il pour s'exprimer plus librement que d'autres ou peut-être, tout simplement, avait-il un tempérament peu enclin à se taire devant les abus. Est-ce là la raison des problèmes qu'il avait déjà eus avec les autorités académiques avant de s'établir à Huelva? L'une des choses que l'on apprend dans la plainte rédigée par le directeur du lycée de Huelva, c'est que, quand Orfois occupait un poste de professeur au lycée de Monforte, il a été puni d'une affectation d'office à celui de Huesca. Mais nous ne connaissons pas les raisons de cette mesure ni celles qui expliquent qu'Orfois ait fini par quitter Huesca, ville située aux pieds des Pyrénées, pour venir quémander un poste de français à Huelva, c'est-à-dire à l'autre bout de l'Espagne.

\subsection{Orfois appuyé par les autorités de Huelva}

Nous venons de résumer le contenu du document rédigé par Orfois en sa défense; il nous reste à parler des trois attestations dont il l'a accompagné. Le simple fait de les avoir sollicitées prouve, à notre avis, qu'Orfois s'est plus ou moins aperçu que c'était l'adéquation de son comportement aux modes de vie d'une ville de province qui était vraiment en jeu dans cette affaire.

Orfois a joint à sa déclaration trois attestations de bonne conduite signées, respectivement, par la plus haute autorité municipale - le maire -, par la plus haute autorité ecclésiastique de la ville - le prêtre de la paroisse principale - et par le délégué du Ministère d'Économie, certainement choisi par Orfois du fait que c'était justement le délégué précédent qui avait dénoncé l'avoir surpris en état d'ivresse. Évidemment, si Orfois a envoyé au recteur leurs attestations, c'est parce que toutes trois soutenaient que son comportement public était excellent. Le délégué le dit laconiquement mais avec force: "[J. Orfois] présente une conduite et jouit d'une réputation irréprochables ». Le maire, en revanche, se montre plus explicite :

J'atteste que $M$. Joseph Orfois, professeur en chair du lycée départemental d'enseignement secondaire, a observé, depuis qu'il réside dans cette ville, une conduite morale, politique et religieuse irréprochable ; jamais, sous aucun rapport, il n'a manqué de garder la dignité la plus stricte dans tous ses actes, raison pour laquelle il a toujours mérité le plus grand respect et la plus haute considération aussi bien de la part des autorités que des particuliers. Parfois, il a été vu s'occuper de l'assistance aux malades sans ressources et de l'instruction religieuse des détenus $[. .$. 
Dans l'attestation du prêtre, enfin, on trouve la confirmation qu'Orfois pratiquait sa religion avec un zèle hors du commun ; mais, contrairement au directeur, le curé n'y voit aucun motif pour l'accuser d'extravagance :

Quoique je n'aie eu que peu de rapports avec lui, je connais M. Joseph Orfois depuis son arrivée au lycée de cette ville. Je n'ai jamais trouvé quoi que ce soit à lui reprocher dans sa conduite morale ou politique, publique ou privée; bien au contraire, il a toujours donné l'exemple au peuple, car je l'ai vu implorer la charité publique pour secourir les pauvres et en distribuer ensuite les aumônes avec la plus grande équité, comme j'ai pu le constater. Je l'ai vu, de même, assister avec son fils aux offices religieux avec la meilleure dévotion, tous deux pratiquant la confession et la communion. Et je l'ai vu aussi, un cierge à la main, accompagner habituellement le Seigneur lorsqu'Il est amené aux malades, moi-même ayant été témoin des reproches qu'il adressait à ceux qui n'observaient pas le recueillement obligé [...].

\section{Le verdict}

31 La commission créée par le Conseil universitaire émet enfin son rapport le 14 août 1867, presque deux ans après le début de l'affaire. Elle en conseille le classement, "étant donné que les charges n'ont pu être complètement prouvées ». Elle signale, cependant, que le document présenté par Orfois en sa défense ne permet pas non plus d'écarter tout à fait la possibilité du bien-fondé de certaines des accusations lancées par le directeur ; de fait, le professeur lui-même, dit-elle, « fait aveu de quelque chose ».

La position de la commission universitaire coïncide donc en grande partie avec celle qu'avait adoptée la commission du Conseil d'instruction de Huelva; la seule mesure qu'elle recommande de prendre est en faite très similaire à celle que ce conseil proposait dans son premier rapport :

[...] enjoindre M. Orfois à s'efforcer de toujours garder la correction et la dignité convenables aussi bien dans son apparence extérieure que dans sa conduite publique. Il doit également renoncer à entrer dans les tavernes et les maisons qui ne soient pas fréquentées par les personnes de la bonne société, ne serait-ce que pour y acheter du vin: ceux qui le voient y accéder ne connaissent pas son intention et ces lieux ne sont pas compatibles avec la retenue et la dignité qui doivent être l'ornement des professeurs, consacrés à la noble mission de l'enseignement.

Comme on le voit, la commission universitaire ne s'est finalement prononcée que sur l'image publique offerte par le professeur, c'est-à-dire sur l'aspect de la question qui a occupé aussi la place centrale dans les rapports des autres instances concernées. Le primordial pour tous, nous semble-t-il, c'était de veiller sur le prestige du corps professoral pour sauvegarder ainsi la position des professeurs parmi les classes moyennes.

\section{Conclusions}

Jusqu'ici l'affaire Orfois. À notre avis, elle révèle les conditions pénibles qu'ont dû endurer un grand nombre de professeurs de l'époque, et tout particulièrement les professeurs de langues étrangères. Comme nous l'avons vu, ce n'est pas l'activité pédagogique d'Orfois qui a été la cause première de la plainte du directeur du lycée. Il nous semble qu'il y avait un mal de fond: la situation économique précaire du 
professeur de français. Très probablement, l'inimitié entre le directeur et Orfois est née du malaise éprouvé par ce dernier du fait de ne pouvoir obtenir une charge horaire plus étendue afin d'augmenter ses revenus, inférieurs à ceux des autres catedráticos du lycée. Et, lorsque la colère du directeur s'est finalement déchaînée contre lui, le problème posé était son exclusion des listes électorales, due aussi à son bas salaire. Si Orfois se démène pour pouvoir voter, ce n'est en réalité qu'afin de se voir reconnu comme membre de l'élite sociale de la ville. Les seuls reproches, enfin, que décident de lui faire les commissions qui ont étudié l'affaire tiennent également à l'insuffisance des moyens économiques du professeur. Orfois lui-même admet qu'il va chercher un café ou un verre de vin dans une épicerie ou une taverne parce qu'il ne peut se permettre de fréquenter les lieux où se réunit la bonne société de Huelva. Tout compte fait, ce qui importe le plus, ce n'est pas qu'il boive ou non en excès, mais le fait qu'on puisse le voir entrer dans des établissements qui, abandonnés aux couches populaires, sont devenus, aux yeux de la classe moyenne, les foyers de l'alcoolisme.

Heureusement pour Orfois, il semble avoir été un catholique pratiquant; mais, curieusement, ses pratiques religieuses tombent dans l'ostentation, ce qui nous amène à nous demander s'il ne chercherait en réalité par là qu'une simple voie pour être admis dans le sein de la société aisée d'une ville de province. Il se peut, pourtant, que, à moyen terme, cette voie ait également fini par lui porter préjudice. En 1868 commence en Espagne la période connue comme le sexenio revolucionario, dont les différents régimes successifs se sont caractérisés par leur belligérance contre le pouvoir de l'Église. Nous avons vu comment les pratiques religieuses d'Orfois l'ont aidé, en 1867, à obtenir de très bonnes attestations, aussi bien de la part du prêtre que de la part du maire. L'attitude des nouvelles autorités révolutionnaires n'a donc pu lui servir d'appui juste au moment où la situation des professeurs de français est devenue encore plus précaire qu'auparavant du fait de la suppression de l'étude des langues étrangères dans l'enseignement secondaire. Certes, en ce qui concerne la situation politique, il y aura un retour à l'état précédent avec le coup d'état contre la première république espagnole et avec la restauration des Bourbons à partir de 1875. Mais Orfois était alors âgé de 57 ou 58 ans. On aimerait savoir dans quelle mesure tous ces bouleversements politicosociaux ont eu une influence dans le statut social et professionnel d'Orfois et des professeurs de français en général. Il est probable que leur sort n'a pas a été le même partout ni pour tous. Mais ces recherches dépassent le cadre étroit de cette étude.

Nous lancerons, toutefois, une invitation. Les archives historiques des universités et des lycées conservent une masse de documents susceptibles de fournir beaucoup de renseignements, non seulement sur l'activité proprement professionnelle des enseignants de langues étrangères, mais également sur leur vie quotidienne et sur les modalités de leur insertion sociale. On dispose déjà d'un grand nombre d'études sur les méthodes d'enseignement qu'ils suivaient, sur leurs possibilités de promotion administrative, sur leurs revenus... Mais, sauf pour des cas spécialement significatifs", on s'est moins souvent occupé de découvrir des détails précis sur leur mode de vie, sur leurs lieux de résidence, sur leur activité mondaine, sur leurs idées politiques et, en général, sur tout ce que l'on ne trouve ni dans les lois et décrets relatifs au monde éducatif ni dans les manuels. Cette information dort pourtant dans les archives, dans des documents manuscrits qui, quoique moins faciles à consulter que les dispositions officielles imprimées, réservent, en revanche, à qui veut les feuilleter des données 
pleines d'intérêt. Il s'agit là d'un cumul d'information qui nous attend. Espérons que leur attente ne sera pas longue.

\section{BIBLIOGRAPHIE}

\section{Bibliographie}

BRUÑA CUEVAS, Manuel (2001), «L'enseignement du français mis au service du régime de Franco (1936-1940) », in Kok Escalle \& Melka (dir.), 303-323.

CORCUERA, Fidel, GASPAR, Antonio (1999), La lengua francesa en España en el siglo XVI. Estudio y edición del Vocabulario de los vocablos de Jacques de Liaño, Saragosse, Prensas Universitarias.

FERNÁNDEZ FRAILE, María Eugenia et SUSO LÓPEZ, Javier (1999), La enseñanza del francés en España (1767-1936), Grenada, Método Ed.

JULIÁ, Santos (2003), « Matar la revolución, construir el Estado : la época moderada (1844-1868) », in J. Valdeón, J. Pérez \& S. Juliá (dir.), Historia de España, Madrid, Espasa Calpe, 355-384.

KOK ESCALLE, Marie-Christine et MELKA, Francine (dir.) (2001), Changements politiques et statut des langues : Histoire et Épistémologie 1780-1945, Amsterdam / Atlanta, Rodopi.

LÉPINETTE, Brigitte (2000), L'enseignement du français en Espagne au XVIII e siècle dans ses grammaires, Münster, Nodus.

LESELBAUM, Charles (1990), « Antonio Machado y la enseñanza del francés en su tiempo », in J. Urrutia (dir.), Antonio Machado hoy, Séville, Alfar, III, 89-107.

MOREU HUET, Núria (1990), Pierre-Nicolas Chantreau (1741-1808) et sa grammaire, Barcelone, P.U.B. ROIG, Carmen (1991), « Père Joseph Núñez de Prado (1666-1743) », Lettre de la SIHFLES 10, 5-6. RUEDA, Germán (1996), El reinado de Isabel II. La España liberal, in J. Manga et al. (dir.), Historia de España 22, Madrid, Historia 16.

SECO, Manuel (1987), « Ramón Joaquín Domínguez », in M. Seco (1987), Estudios de lexicografía española, Madrid, Paraninfo, 152-164. .

SERRANO, Montserrat, AVENDAÑO, Lina, MOLINA, María del Carmen dir. (2000), La Philologie française à la croisée de l'an 2000, Grenade, APFFUE, Universidad de Granada

SUÁREZ GÓMEZ, Gonzalo (1956), La enseñanza del francés en España (comentario a una bibliografía establecida hasta 1850), Madrid, Universidad Complutense de Madrid (thèse inédite).

SUPIOT, Alberto (1995), « Heurs et malheurs d'un professeur de français dans l'Espagne de la fin du XVIII ${ }^{e}$ siècle : Jean Thomas Lespardat ", Documents pour l'histoire du français langue étrangère ou seconde 16, 7-22.

SUSO LÓPEZ, Javier (2000), « Semblanza de Enrique Canito : afrancesado, francófilo, filántropo », in M. Serrano, L. Avendaño \& Mª.C. Molina (dir.), II, 331-340. 


\section{NOTES}

1. Les documents relatifs à cette affaire constituent le dossier L 648 (19) des Archives historiques de l'Université de Séville.

2. À l'époque, en Espagne comme ailleurs en Europe, le système électoral était censitaire : seuls ceux qui dépassaient un certain niveau de revenus ou ceux qui exerçaient certaines professions libérales avaient le droit de vote. Le nombre d'électeurs n'étant donc pas excessif, parmi les fonctions principales du préfet (gobernador civil) de chaque département se trouvait celle d'assurer au gouvernement en place sa victoire aux élections (Julià, 2003 : 359).

3. Au moment où se situe l'affaire Orfois, le Ministère d'Économie (Ministerio de Fomento) était chargé de l'enseignement. Il est donc très significatif que ce soit précisément le délégué départemental de ce ministère qui ait dénoncé le professeur de français.

4. Parmi les études consacrées à la vie de certains professeurs de français exerçant leur activité en Espagne peuvent être cités les renseignements biographiques donnés par Suárez Gómez (1956) pour un bon nombre d'entre eux ou ceux donnés par Lépinette (2000) pour des auteurs de grammaires du XVIIIe siècle. Roig (1991) fait une première approche biographique de José Núñez de Prado, Bruña Cuevas (2001) la fait de Mario Mirmán et Moreu Huet raconte dans sa thèse (1990) la vie presque romanesque de Pierre-Nicolas Chantreau. Nous trouvons admirables les recherches d'archives sur la vie de Jacques Ledel réalisées par Corcuera \& Gaspar (1999), celles de Seco (1987) sur Ramón Joaquín Domínguez et celles de Leselbaum (1990) sur l'activité comme professeur de français du grand poète Antonio Machado. Citons enfin, last but not least, les très intéressantes études consacrées à Jean Thomas Lespardat par Supiot (1995) et à Enrique Canito par Suso (2000).

\section{RÉSUMÉS}

En 1865, le directeur du lycée de Huelva, petite ville andalouse, adresse au recteur de l'université de Séville, dont dépendait le lycée, une pétition de sanction pour le professeur de français de son établissement. Les raisons présentées par le directeur pour sa demande, ainsi que la série de documents auxquels elle donnera lieu, tracent un profil détaillé et savoureux de ce qu'on attendait à l'époque d'un professeur de lycée, aussi bien en tant qu'enseignant qu'en tant qu'individu appartenant à la petite bourgeoisie locale.

In 1865 , the headmaster of the secondary school of Huelva - a small Andalousian town addressed a request to the rector of the University of Sevilla, on which the secondary school was dependent, to take action against the French teacher of his establishment. The reasons presented by the headmaster for his request and the documents it would generate, sketch the detailed and juicy profile of the expectations about a secondary school professor at the time, not only as a teacher but also as a local middle-class person. 
INDEX

Mots-clés : compétences linguistiques et didactiques, professeur, disciplinarisation, Espagne, formation, institutionnalisation, profil professionnel, XIXe siècle

Keywords : disciplinarisation, teacher, institutionnalisation, linguistic and paedagogical competences, professional profile, Spain, XIXth century

\section{AUTEUR}

\section{MANUEL BRUÑA CUEVAS}

Universidad de Sevilla 